\title{
Improved Conditions for the Existence and Uniqueness of Solutions to the General Equality Constrained Quadratic Programming Problem
}

\author{
Amadu Fullah Kamara ${ }^{1,2^{*}}$, Mohamed Abdulai Koroma ${ }^{2,3}$, Mujahid Abd Elmjed M.-Ali ${ }^{1,4}$ \\ ${ }^{1}$ Department of Mathematics, University of Science and Technology of China, Hefei, China \\ ${ }^{2}$ Department of Mathematics, Faculty of Pure and Applied Sciences, Fourah Bay College, \\ University of Sierra Leone, Freetown, Sierra Leone \\ ${ }^{3}$ Department of Mathematics, Harbin Institute of Technology, Harbin, China \\ ${ }^{4}$ Department of Mathematics, Faculty of Education, Kassala University, Kassala, Sudan \\ Email: *amadu_fullah2005@yahoo.com,d2ydx@yahoo.com,mujahid@mail.ustc.edu.cn
}

Received October 21, 2012; revised November 24, 2012; accepted December 3, 2012

\begin{abstract}
This paper presents an approach that directly utilizes the Hessian matrix to investigate the existence and uniqueness of global solutions for the ECQP problem. The novel features of this proposed algorithm are its uniqueness and faster rate of convergence to the solution. The merit of this algorithm is base on cost, accuracy and number of operations.
\end{abstract}

Keywords: Hessian Matrix; Global Solutions; Equality Constrained Quadratic Programming; Existence and Uniqueness of Solutions; Lagrangian Methods; Schur Complement Methods

\section{Introduction}

Usually the general quadratic programming (GQP) problem has a structure of the form

$$
\begin{gathered}
G Q P: \min _{x} q(x)=\frac{1}{2} x^{\mathrm{T}} G x+x^{\mathrm{T}} c \\
\text { subject to } \quad a_{i}^{\mathrm{T}} x=b_{i}, \quad i \in \varepsilon, \\
a_{i}^{\mathrm{T}} x \geq b_{i}, \quad i \in \Omega .
\end{gathered}
$$

where $G$ is a symmetric $n \times n$ matrix, $\varepsilon$ and $\Omega$ are finite sets of indices. In quadratic programming problems, the matrix $G$ is called the Hessian matrix. The vectors $c, x$ and $a_{i}$ are column vectors in $\mathbb{R}^{n}$. To make computational life easier, we consider only the equality constraints and formulate the equality constrained quadratic programming (ECQP) problem as follows

$$
\begin{gathered}
\text { ECQP : } \min _{x} q(x)=\frac{1}{2} x^{\mathrm{T}} G x+x^{\mathrm{T}} C \\
\text { subject to } A x=b
\end{gathered}
$$

where $A$ is a $m \times n$ jacobian matrix of constraints (with $m \leq n$ ) [1]. Throughout this paper, we will assume that $A$ can be of any form, since it is not a par-

\footnotetext{
"Corresponding author.
}

ticipant in the determination of the ECQP's global minimum. Quadratic programming problems occur naturally, and sometimes stem as subproblems in general constrained optimization methods, such as sequential quadratic programming, augmented Lagrangian methods, and interior point methods. This type of programming problems occurs in almost every discipline and as a result became a topic of interest to a lot of researchers [1-3].

In sequential quadratic programming (SQP) algorithms, an ECQP phase that employs second derivative information (Hessian matrix) is usually added to enhance rapid convergence to the solution [4-7] SQP algorithms [8] that utilize the exact Hessian matrix are often preferred to those that use convex quasi-Newton approximations [9-11] since they need lesser time to converge to the solution.

In 1985, Gould investigates the conditions under which the ECQP problem can be said to have a finite solution. Gould's analysis of the ECQP problem is based on the concepts of the reduced Hessian matrix $\left(Z^{\mathrm{T}} G Z\right)$ and signs of the eigenvalues of the Karush-Kuhn Tucker (KKT) matrix [3]. The well known $(K K T)$ matrix has the form

$$
K=\left(\begin{array}{cc}
G & A^{\mathrm{T}} \\
A & 0
\end{array}\right)
$$


[1]. For small-scale ECQP problems it is possible to solve the KKT matrix (and hence, the ECQP problem ) analytically $[1,3,12]$. The matrix $Z$ is one whose columns are a basis for the null space of $A$ (matrix of contraints), and is obtained from the $Q R$ factorization of $A$. We investigated the method and found that the reduced Hessian matrix is not always accurate due to rounding off errors arising in the calculation of $Z$ [13-15].

Our goal in this paper is to present a new method that utilizes a necessary and sufficient condition for the existence and uniqueness of the solutions of the ECQP problem. In this paper, we show that for the ECQP problem to have a global solution, its Hessian matrix must possess a Cholesky factor. As we shall see in Section 2, this paper focuses only on the condition(s) under which the ECQP problem is said to have a global solution [16].

This paper is organized as follows. In Section 2, we discuss our method. Gould's method is reviewed in Section 3. The analysis follow in Section 4 and some concluding remarks are made in Section 5.

\section{Method}

In this section, we introduce our new method of analyzing the solution of the ECQP problem. It is based on the fact that the Cholesky decomposition is unique for positive definite matrices.

\section{Cholesky Decomposition}

Let $M \in \mathbb{R}^{n \times n}$ be a matrix that can undergo Cholesky decomposition with a Cholesky factor $L$ (Lower triangular matrix) then we can write

$$
M=L \times L^{\mathrm{T}}
$$

where $L^{\mathrm{T}}$ is the transpose of $L$. We let

$$
L=\left(\begin{array}{cc}
s & 0 \\
\beta & \alpha
\end{array}\right) .
$$

Substituting Equation (2.2) into Equation (2.1) gives

$$
M=\left(\begin{array}{cc}
s & 0 \\
\beta & \alpha
\end{array}\right)\left(\begin{array}{cc}
s & \beta \\
0 & \alpha
\end{array}\right)=\left(\begin{array}{cc}
s^{2} & s \beta \\
s \beta & \alpha^{2}+\beta^{2}
\end{array}\right)
$$

From Equation (2.3), we see that the conditions for $M$ to be positive definite are satisfied. Therefore,our conditions for positive definiteness are; the matrix must be a square matrix and possesses a Cholesky factor.

We let $X$ to be a $2 \times 1$ column vector say

$$
X=\left(\begin{array}{l}
x_{1} \\
x_{2}
\end{array}\right)
$$

and we write

$$
\begin{aligned}
X^{\mathrm{T}} M X & =\left(\begin{array}{ll}
x_{1} & x_{2}
\end{array}\right)\left(\begin{array}{cc}
s^{2} & s \beta \\
s \beta & \alpha^{2}+\beta^{2}
\end{array}\right)\left(\begin{array}{l}
x_{1} \\
x_{2}
\end{array}\right) \\
& =s^{2} x_{1}^{2}+\left(\alpha^{2}+\beta^{2}\right) x_{2}^{2}+2 s \beta x_{1} x_{2}
\end{aligned}
$$

From Equation (2.3), it is clear that the first and second terms are always positive, which implies their sum is also always positive and greater than the third term if $x_{1} \neq x_{2} \neq 0$. When $x_{1}=x_{2}=0$, the matrix $X^{\mathrm{T}} M X$ always equals zero. Therefore, the matrix $X^{\mathrm{T}} M X$ is always positive if and only if the column vector $X$ has entries $x_{1}$ and $x_{2}$ (such that $x_{1} \neq x_{2} \neq 0$ ) and the matrix $M$ has a Cholesky factor.

In the above demonstration $n=2$, which means that a $2 \times 2$ matrix $M$ and a $2 \times 1$ column vector $X$ produces Equation (2.5). Analogously, any $n \times n$ matrix $M$ and any $n \times 1$ column vector $X$ (where $n \geq 2$ ) shall produce an equation similar in properties to Equation (2.5). If and only if $x_{1} \neq x_{2} \neq x_{3} \neq \cdots \neq x_{n} \neq 0$.

Corollary 2.1: Let $A$ be any non-singular matrix and the Hessian matrix being Cholesky factorizable. Then the KKT matrix

$$
K=\left(\begin{array}{cc}
G & A^{\mathrm{T}} \\
A & 0
\end{array}\right)
$$

is nonsingular and has a unique solution.

Corollary 2.2: Let $K$ be the Karush-Kuhn-Tucker matrix

$$
K=\left(\begin{array}{cc}
G & A^{\mathrm{T}} \\
A & 0
\end{array}\right)
$$

and assume $A$ is any matrix. Then the ECQP problem has a global minimum if and only if the Hessian matrix has a Cholesky factor.

\section{Review of Gould's Method}

In this section, we review Gould's method. The method consists of three approaches: Null-space methods, Lagrangian methods and Schur complement methods [12].

Null-space methods: For $x^{*}$ to be a solution of the ECQP problem, a vector $\lambda^{*}$ (i.e. Lagrange multipliers) must exist such that the system of equations below is satisfied

$$
\left(\begin{array}{cc}
G & -A^{\mathrm{T}} \\
A & 0
\end{array}\right)\left(\begin{array}{c}
x^{*} \\
\lambda^{*}
\end{array}\right)=\left(\begin{array}{c}
-c \\
b
\end{array}\right) .
$$

We let

$$
x^{*}=x+p,
$$

with $x$ being some estimate of the solution and $p$ the desired step. By expressing $x^{*}$ as in Equation (3.2), Equation (3.1) can be written in a form that is more useful for computational purposes as given below 


$$
\left(\begin{array}{cc}
G & A^{\mathrm{T}} \\
A & 0
\end{array}\right)\left(\begin{array}{c}
-p \\
\lambda^{*}
\end{array}\right)=\left(\begin{array}{l}
g \\
h
\end{array}\right)
$$

where

$$
\begin{gathered}
h=A x-b, \\
g=c+G x, \\
p=x^{*}-x .
\end{gathered}
$$

This method finds $p$ and $\lambda^{*}$ first, by partitioning the vector $p$ into two components as follows

$$
p=Y p_{Y}+Z p_{Z},
$$

where $Y$ and $Z$ have orthonormal columns and can be obtain from the $Q R$ factorization of $A$. An interesting property of this approach is that $A Z=0 \quad[1,3]$, which makes the calculation of $p$ and $\lambda^{*}$ possible by solving the four equations below

$$
\begin{gathered}
(A Y) p_{Z}=-h, \\
-G Y p_{Y}-G Z p_{Z}+A^{\mathrm{T}} \lambda^{*}=g, \\
\left(Z^{\mathrm{T}} G Z\right) p_{Z}=-Z^{\mathrm{T}} G Y p_{Y}-Z^{\mathrm{T}} g, \\
(A Y)^{\mathrm{T}} \lambda^{*}=Y^{\mathrm{T}}(g+G p) .
\end{gathered}
$$

This method has a wider application than the Rangespace methods because; it doesn't require $G$ being nonsingular. According to this paper, the condition, that $G$ must undergo Cholesky decomposition is the only requirement for the ECQP problem to have a global minimum. A knowledge of the null space basis matrix $Z$ is not important at all.

Lagrangian methods: This method calculates the values of $p$ and $\lambda^{*}$ directly from Equation (3.3), i.e. the Karush-Kuhn-Tucker equations for the ECQP problem.

In this paper, the ECQP problem can only have a global minimum if $G$ possesses a Cholesky factor (Corollary 2.1,2.2) .

Schur complement methods: Here we assume that $G$ has a Cholesky factor and derive two equations from Equation (3.3) for the solutions of $p$ and $\lambda^{*}$. These equations are as follows

$$
\begin{gathered}
\left(A G^{-1} A^{\mathrm{T}}\right) \lambda^{*}=\left(A G^{-1} g-h\right), \\
G p=A^{\mathrm{T}} \lambda^{*}-g .
\end{gathered}
$$

It is easy to see that both $G$ and $G^{-1}$ are positive definite. In this paper, we show that $G$ and $G^{-1}$ have Cholesky factors and hence $A G^{-1} A^{\mathrm{T}}$ is always positive definite, which indicate the existence of a global solution for the ECQP problem Section 2.

\section{Analysis}

In this section we will solve a numerical example from [1] using our algorithm and compared our results with those of Gould's method. Let us consider the ECQP problem below and deduce whether it has a global minimum or not by using Gould's method and our algorithm.

$$
\begin{aligned}
& \min q(x)=3 x_{1}^{2}+2 x_{1} x_{2}+x_{1} x_{3}+2.5 x_{2}^{2} \\
& +2 x_{2} x_{3}+2 x_{3}^{2}-8 x_{1}-3 x_{2}-3 x_{3}, \\
& \text { subject to } \quad x_{1}+x_{3}=3, \quad x_{2}+x_{3}=0 .
\end{aligned}
$$

We will write the above ECQP problem in the standard form described in the introduction by defining

$$
\begin{gathered}
G=\left(\begin{array}{lll}
6 & 2 & 1 \\
2 & 5 & 2 \\
1 & 2 & 4
\end{array}\right), \quad c=\left(\begin{array}{l}
-8 \\
-3 \\
-3
\end{array}\right), \\
A=\left(\begin{array}{lll}
1 & 0 & 1 \\
0 & 1 & 1
\end{array}\right), \quad b=\left(\begin{array}{l}
3 \\
0
\end{array}\right) .
\end{gathered}
$$

For Gould's algorithm we need to find $Z$ from the $Q R$ factorization of matrix $A$ i.e. $A=Q R$.

$$
\begin{gathered}
Q=\left(\begin{array}{ccc}
0.7071 & -0.4082 & -0.5774 \\
0 & 0.8165 & -0.5774 \\
0.7071 & 0.4082 & 0.5774
\end{array}\right), \\
R=\left(\begin{array}{cc}
1.4142 & 0.7071 \\
0 & 1.2247 \\
0 & 0
\end{array}\right) .
\end{gathered}
$$

We can obtain $Z$ from the column space of matrix $Q$ and the matrix $Z$ must satisfies the constrain $A Z=0$. Hence we have

$$
Z=\left(\begin{array}{c}
-0.5774 \\
-0.5774 \\
0.5774
\end{array}\right) .
$$

Therefore, $\quad Z^{\mathrm{T}} G Z=4.3341>0$ and according to Gould's algorithm the ECQP problem has a global minimum.

For our algorithm we only need to show that the matrix $G$ has a Cholesky factor. Let $L$ be the Cholesky factor of $G$.

$$
L=\left(\begin{array}{ccc}
2.4495 & 0 & 0 \\
0.8165 & 2.0817 & 0 \\
0.4082 & 0.8006 & 1.7867
\end{array}\right)
$$

According to our algorithm,this implies the matrix $G$ is positive definite and therefore the ECQP problem has a minimum solution. To show this fact we select any matrix that is a subset of the set of matrices described in subsection (2.1) and suppose we have that matrix to be $P=\left(\begin{array}{lll}-8 & -3 & -3\end{array}\right)$, then $P^{\mathrm{T}} G P=645>0$. 
Let us consider another matrix

$$
Q=\left(\begin{array}{ll}
4 & 8 \\
1 & 0 \\
3 & 2
\end{array}\right) .
$$

We will have result

$$
Q^{\mathrm{T}} G Q=\left(\begin{array}{cc}
189 & 268 \\
268 & 432
\end{array}\right)>0 .
$$

Finally, we consider a matrix with all negative entries as follows

$$
K=\left(\begin{array}{cc}
-4 & -8 \\
-1 & 0 \\
-3 & -2
\end{array}\right) .
$$

This gives the result

$$
K^{\mathrm{T}} G K=\left(\begin{array}{ll}
189 & 268 \\
268 & 432
\end{array}\right)>0 .
$$

From the above example, we observed the following result:

1) Multiplying a matrix $G$ that has a Cholesky factor with any other matrix except the zero matrix, doesn't alter the positive definite property of matrix $G$ and hence the existence of global minimum.

2) Decimals are encountered in Gould's approach which may lead to rounding off errors and hence inaccuracy. Decimals have no effects on our method as long as the Hessian matrix has a Cholesky factor.

3) The number of matrix operations that are involved in Gould's approach are far more than those that are involved in our algorithm which implies that our method is faster than that of Gould.

Gould's approach uses the notion of the reduced Hessian matrix and the signs of the eigenvalues of the Karush-Kuhn-Tucker matrix to analyze the conditions under which the ECQP problem shall have a global solution [3]. It is clear that $Z^{\mathrm{T}} G Z$ is sometimes incorrect due to rounding off errors in the calculation of $Z$. In this paper, we present a method that directly utilizes the Hessian matrix to analyze global minimum conditions for the ECQP problem.

Finally, this proposed method has fewer iterations than Gould's algorithm, inexpensive and naturally faster (Cholesky factorization) than Gould's approach (with more iterations).

\section{Conclusions}

In 1985, Gould investigates the practical conditions for the existence and uniqueness of solutions of the ECQP problem based on $Z^{\mathrm{T}} G Z$ and inertia of the $K K T$ matrix. In this piece of work, we present a new method that directly works with $G$ to analyze global solutions of the ECQP problem.

The advantages of our method lie in its accuracy, cost and number of operations. It is true that this noble algorithm is unique and computationally faster (i.e. Cholesky decomposition) than Gould's method. Our method also revealed that if the Hessian matrix has a Cholesky factor then, the Hadamard inequality [17] for positive definiteness is satisfied as well.

We finally conclude that the existence and uniqueness of solutions of the ECQP problem is independent of its constraints but depend wholly and solely on the Hessian matrix $(G)$.

\section{Acknowledgements}

We would like to thank Khalid O. Elaalim for his useful contributions during the early stages of this work. We are also grateful to anonymous referees for their valuable comments on this paper. Finally, we would like to extend special thanks to the Chinese Scholarship Council which funded this research.

\section{REFERENCES}

[1] J. Nocedal and S. J. Wright, "Numerical Optimization," 2nd Edition, Springer Series in Operations Research and Financial Engineering, Springer, New York, 2006, pp. 448-492. doi:10.1007/978-0-387-40065-5_16

[2] N. I. M. Gould, M. E. Hribar and J. Nocedal, "On the Solution of Equality Constrained Quadratic Programming Problems Arising in Optimization," SIAM Journal on Scientific Computing, Vol. 23, No. 4, 2001, pp. 1376-1395. doi:10.1137/S1064827598345667

[3] N. I. M. Gould, “On Practical Conditions for the Existence and Uniqueness of Solutions to the General Equality Quadratic Programming Problem,” Mathematical Programming, Vol. 32, No. 1, 1985, pp. 90-99. doi:10.1007/BF01585660

[4] R. H. Byrd, N. I. M. Gould, J. Nocedal and R. A. Waltz, "An Algorithm for Nonlinear Optimization Using Linear Programming and Equality Constrained Subproblems," Mathematical Programming, Vol. 100, No. 1, 2004, pp. 27-48.

[5] N. I. M. Gould and D. P. Robinson, "A Second Derivative SQP Method: Global Convergence,” SIAM Journal on Optimization, Vol. 20, No. 4, 2010, pp. 2023-2048. doi:10.1137/080744542

[6] N. I. M. Gould and D. P. Robinson, “A Second Derivative SQP Method: Local Convergence and Practical Issues,” SIAM Journal on Optimization, Vol. 20, 2010, pp. 2049-2079. doi:10.1137/080744554

[7] J. L. Morales, J. Nocedal and Y. Wu, "A Sequential Quadratic Programming Algorithm with an Additional Equality Constrained Phase," IMA Journal of Numerical Analysis, Vol. 32, No. 2, 2012, pp. 553-579. doi:10.1093/imanum/drq037 
[8] R. Fletcher and S. Leyffer, "Nonlinear Programming without a Penalty Function," Mathematical Programming, Vol. 91, No. 2, 2002, pp. 239-269. doi:10.1007/s101070100244

[9] A. Drud, "CONOPT: A GRG Code for Large Sparse Dynamic Nonlinear Optimization Problems,” Mathematical Programming, Vol. 31, No. 2, 1985, pp. 153-191. doi:10.1007/BF02591747

[10] P. E. Gill, W. Murray and M. A. Saunders, "SNOPT: An SQP Algorithm for Large-Scale Constrained Optimization,” SIAM Journal on Optimization, Vol. 12, No. 4, 2002, pp. 979-1006. doi:10.1137/S1052623499350013

[11] K. Schittkowski, “The Nonlinear Programming Method of Wilson, Han and Powell with an Augmented Lagrangian Type Line Search Function,” Numerische Mathematik, Vol. 38, No. 1, 1981, pp. 83-114. doi:10.1007/BF01395810

[12] S. Boyd and L. Vandenberghe, "Convex Optimization," Cambridge University Press, Cambridge, 2004, pp. 241-
245.

[13] E. Anderson, Z. Bai and J. Dongarra, "Generalized QR Factorization and Its Application,” Linear Algebra and Its Applications, Vol. 162-164, 1992, pp. 243-271. doi:10.1016/0024-3795(92)90379-O

[14] P. E. Gill and W. Murray, "Numerically Stable Methods for Quadratic Programming," Mathematical Programming, Vol. 14, No. 1, 1978, pp. 349-372. doi:10.1007/BF01588976

[15] M. OCW, “Positive Definite Matrices and Minima,” 2012. http://ocw.mit.edu/courses/mathematics/18-06sc-linear-al gebra-fall-2011/positive-definite-matrices-and -applications/postive-definite-matrices-and-minima/MIT1 8_06SCF11_Ses3.3prob.pdf

[16] Wikipidia, "Positive-Definite Matrix,” 2012. http://en.wikipedia.org/wiki/Positive-definite_matrix

[17] R. A. Horn and C. R. Johnson, “Matrix Analysis,” Cambridge University Press, Cambridge, 1985. 